\title{
HELICOBACTER PYLORI E DOENÇA PÉPTICA. Estudo comparativo de métodos diagnósticos
}

\author{
Alaor CAETANO'1, Valter Nilton FELIX², \\ Fernando Tadeu Vanucci COIMBRA ${ }^{1}$ e Arnaldo José GANC ${ }^{3}$
}

\begin{abstract}
RESUMO - Foram estudados, prospectivamente, 150 pacientes. Estudo endoscópico revelou gastrite crônica em 109 pacientes (72,6\%), úlcera gástrica em 6 (4\%), duodenite crônica em 9 (6\%) e úlcera duodenal em $26(17,4 \%)$. Quanto à avaliação metodológica para pesquisa do Helicobacter pylori, $103(68,67 \%)$ apresentaram teste da urease positivo, $104(69,33 \%)$, positividade histopatológica e 98 (65,33\%), positividade sorológica. Não houve diferença estatística entre os métodos. Pela facilidade de realização, o teste da urease credencia-se como o de melhor indicação nos pacientes que também se beneficiarão com o diagnóstico endoscópico. Caso a endoscopia digestiva alta não possa ou não deva ser realizada, está recomendado o teste sorológico.
\end{abstract}

DESCRITORES - Infecções por Helicobacter, diagnóstico. Testes sorológicos. Urease.

\section{INTRODUÇÃO}

A endoscopia digestiva alta, complementada com biopsias da mucosa gástrica, mantém-se como método prevalente na detecção do Helicobacter pylori (H. pylori), no Brasil.

Este estudo teve o objetivo de comparar entre si os testes usuais de pesquisa da bactéria, com base em expressiva casuística.

\section{MÉTODO}

Estudaram-se, prospectivamente, 150 pacientes, de um total de 1.043 atendimentos, por queixas compatíveis com doenças pépticas, no Serviço de Endoscopia Multidisciplinar do Hospital de Base de São José do Rio Preto, SP.

Eram 76 homens e 74 mulheres, o tempo de evolução dos sintomas variava de alguns dias até mais de 20 anos, sendo prevalente o período de 1 a 10 anos.

Foram excluídos pacientes que faziam uso de qualquer medicação com finalidade antiinflamatória ou que apresentavam afecções que cursassem com imunodepressão, tais como tuberculose, blastomicose e AIDS, neoplasias, gastrite atrófica ou qualquer tipo de operação prévia relacionada com o sistema digestório.

Foram ainda excluídos os que relatavam hemorragia digestiva alta, uso de drogas para tratamento de verminose, uso recente de antibióticos ou de qualquer produto para alívio dos sintomas.
O exame endoscópico seguiu os ditames da Sociedade Brasileira de Endoscopia Digestiva.

Jejum mínimo de 12 horas foi solicitado a todos os pacientes e, antes da realização do exame, cada um aceitou ser incluído no protocolo, após pormenorizada explicação do mesmo, assinando termo de consentimento.

Era colhido, com pinça endoscópica, um fragmento da mucosa antral para o teste da urease, sendo transferido, com auxílio de agulha descartável, diretamente ao tubo de ensaio contendo a solução de uréia, fornecido pela Probac do Brasil ${ }^{\circledR}$, e guardado em ambiente próximo a $36^{\circ} \mathrm{C}$. A verificação do resultado era realizada após 6 horas.

Outro fragmento de biopsia antral, para o exame histológico, era depositado em frasco com solução de formalina a $10 \%$ e levado a processo histológico rotineiro, desidratação em álcool, diafanização em xilol, inclusão em parafina, sendo depois submetido a cortes de 5 micra e corados por hematoxilina-eosina e Giemsa.

Eram coletados $20 \mathrm{~mL}$ de sangue para o exame sorológico, que foram centrifugados em tubo de ensaio por 10 minutos, à velocidade de 1.500 a 2.000 rotações $/ \mathrm{min}$, para separação do soro, estocado a $20^{\circ} \mathrm{C}$. A leitura foi feita em bloco, pelo método ELISA, para detecção e quantificação de anticorpos do tipo IgG.

$\mathrm{Na}$ análise estatística dos resultados obtidos, utilizouse o teste do $\chi^{2}$, com $P<0,05$ e intervalo de confiança (IC) de $95 \%$. 


\section{RESULTADOS}

O estudo endoscópico revelou gastrite crônica em 109 pacientes $(72,6 \%)$, úlcera gástrica em $6(4 \%)$, duodenite crônica em $9(6 \%)$ e úlcera duodenal em $26(17,4 \%)$.

De acordo com os achados endoscópicos, foram compilados separadamente, de início, os resultados do teste da urease, da histologia e da sorologia (Tabela 1).

TABELA 1. Positividade de testes diagnósticos do $H$. pylori (\%)

\begin{tabular}{lccc}
\hline Afecção & Teste da urease & Histopatologia & Sorologia \\
\hline Gastrites & 66 & 67,8 & 65,1 \\
Úlcera gástrica & 50 & 66,7 & 66,7 \\
Duodenites & 88,8 & 100 & 66,7 \\
Úlcera duodenal & 77 & 77 & 65,4 \\
Total & 68,6 & 69,3 & 64,6 \\
\hline
\end{tabular}

Posteriormente todos os pacientes foram agrupados, tendo-se que $103(68,67 \%)$ apresentaram teste da urease positivo, 104 $(69,33 \%)$, positividade histológica e $98(65,33 \%)$ positividade sorológica para o H. pylori.

Não se observou diferença estatisticamente significante na comparação entre os métodos diagnósticos, quer na casuística fragmentada em relação à doença, ou na geral.

\section{DISCUSSÃO}

A alta prevalência de infecção pelo $H$. pylori, sua forte tendência à cronicidade, bem como a aceitação de importante papel deste microorganismo na patogênese de úlceras gastroduodenais, câncer gástrico e linfomas MALT sustentam o interesse e a preocupação em relação ao assunto.

Há estudos que revelam correlação entre a infecção pelo H. pylori e alterações anatomopatológicas na mucosa gástrica, não só com respeito à inflamação, que varia em extensão e intensidade, como em relação à atrofia, à metaplasia intestinal e à hiperplasia de folículos linfóides ${ }^{(2)}$.

A distribuição focal ou segmentar do $H$. pylori na superfície da mucosa gástrica pode ser responsável, em parte, pela diferença de sensibilidade dos diversos testes diagnósticos.

Neste estudo, para busca diagnóstica do H. pylori, foram realizadas biopsias da região pré-pilórica, pois o antro é o melhor local para a obtenção de amostras para a pesquisa histológica da infecção, por conter a maior densidade de bactérias.
O teste diagnóstico ideal deve ter grandes sensibilidade e especificidade, baixo custo, fácil realização, uso de equipamento e técnica rotineiros e boa aceitação pelo paciente.

Os métodos invasivos podem ser diretos, buscando demonstração histológica do microrganismo, ou indiretos, dependentes da pesquisa da urease pré-formada, uma das principais características do H. pylori.

O exame histológico oferece a possibilidade de visualização microscópica concomitante da mucosa, com melhor definição da gravidade da inflamação. Hematoxilina-eosina e Giemsa são as técnicas de coloração mais utilizadas, depois da inclusão em parafina, na pesquisa do $H$. pylori, como feito neste estudo.

$\mathrm{O}$ microorganismo produz grande quantidade de urease, que catalisa a degradação da uréia em amônia e bicarbonato, levando a aumento do $\mathrm{pH}$ do meio, o que pode ser detectado através de um indicador de $\mathrm{pH}$, conferindo positividade ao teste.

Há ainda os métodos não invasivos, que não requerem endoscopia e são representados pela sorologia neste estudo. Os anticorpos IgM podem não diferir entre indivíduos atualmente H. pylori-positivos e negativos. Por esta razão, é indicado o emprego de ELISA em busca da dosagem de anticorpos IgG, como foi feito no presente trabalho.

A sorologia de fato mostrou-se eficaz na detecção do H. pylori, com positividade de $65,33 \%$ na casuística geral, equiparandose estatisticamente ao teste da urease e à histologia, resultado semelhante ao de publicação recente ${ }^{(5)}$.

É teste preferencial para alguns, por ser não invasivo e atingir taxas de detecção do H. pylori semelhantes às de métodos que utilizam biopsias ${ }^{(4)}$.

O conhecimento atual de várias cepas do $H$. pylori, de sua capacidade de produção de diferentes citotoxinas, da grande soropositividade de anticorpos anti-CagA, em casos de neoplasia gástrica, os progressos de estudos com base em dados genéticos e moleculares ${ }^{(1,3)}$ fazem prever que brevemente os métodos laboratoriais ganharão terreno, em relação aos invasivos.

No entanto, neste momento, o teste da urease e o exame histológico mantêm lugar de destaque na pesquisa do $H$. pylori, diante da realidade brasileira. Em função disto e ainda considerando facilidade de realização e baixo custo, o teste da urease se credencia como o de melhor indicação nos pacientes que também se beneficiarão com o diagnóstico endoscópico.

A equiparação dos resultados do teste sorológico aos dos exames invasivos abre perspectiva para sua indicação segura, caso a endoscopia digestiva alta não possa ou não deva ser realizada. 
Caetano A, Felix VN, Coimbra FTV, Ganc AJ. Helicobacter pylori and peptic disease. Comparative study of the diagnostic methods. Arq Gastroenterol. 2008;45(3):255-7.

ABSTRACT - Prospective endoscopic study of 150 patients revealed chronic gastritis in 109 (72.6\%), gastric ulcer in 6 (4\%), chronic duodenitis in 9 (6\%) and duodenal ulcer in 26 (17.4\%). Searching for Helicobacter pylori, positive urease test was observed in $103(68.67 \%)$, histologic evidence in 104 (69.33\%) and positive serologic test in $98(65.33 \%)$, without statistical difference. The urease test is recommended in the diary medical practice, for the patients who also will benefit themselves with the endoscopic diagnosis. On the other hand, the serologic test is useful when the endoscopy of the upper digestive tract cannot or must not be realized.

HEADINGS - Helicobacter infections, diagnosis. Serologic tests. Urease.

\section{REFERÊNCIAS}

1. Ladeira MSP, Salvadori DMF, Rodrigues MAM. Biopatologia do Helicobacter pylori. J Bras Patol Med Lab. 2003;39:335-42.

2. Matthews GM, Cummins AG, Lawrence A, Johnson B, Campbell F, Butler RN. 13C urea breath test: reproducibility and association with the severity of Helicobacter pylori associated antral gastritis. J Gastroenterol Hepatol. 2005;20:270-4.

3. Suerbaum M, Michetti P. Helicobacter pylori infection. N Engl J Med 2002;347:1175-86.
4. Thomas R, Sousa JC. Screening for Helicobacter pylori. Rev Port Clin Geral. 2006;22:585-9.

5. Tonelli E, Freire LMS, editores. Doenças infecciosas na infância e adolescência. São Paulo: Medsi; 2000. 